\title{
An Adaptive Momentum CMA Blind Equalization Based on Error Energy
}

\author{
Ying Xiao, Yuhua Dong, Jinyu Sun \\ College of Information and Communication Engineering, Dalian Nationality University, Dalian, China \\ Email: xiaoying@126.com, dongyuhua@126.com,2440452893@qq.com
}

How to cite this paper: Xiao, Y., Dong, Y.H. and Sun, J.Y. (2017) An Adaptive Momentum CMA Blind Equalization Based on Error Energy. Int. J. Communications, Network and System Sciences, 10, 333-340. https://doi.org/10.4236/ijcns.2017.105B033

Received: July 20, 2017

Accepted: July 28, 2017

Published: August 4, 2017

\begin{abstract}
To solve the problem of large steady state residual error of momentum constant modulus algorithm (CMA) blind equalization, a momentum CMA blind equalization controlled by energy steady state was proposed. The energy of the equalizer weights is estimated during the updating process. According to the adaptive filtering theory, the energy of the equalizer weights reaches to the steady state after the algorithm is converged, and then the momentum can be set to 0 when the energy change rate is less than the threshold, which can avoid the additional gradient noise caused by momentum and further improve the convergence precision of the algorithm. The proposed algorithm takes advantage of momentum to quicken the convergence rate and to avoid the local minimum in the cost function to some extent; meanwhile, it has the same convergence precision with CMA. Computer simulation results show that, compared with CMA, momentum CMA (MCMA) and adaptive momentum CMA (AMCMA) blind equalization, the proposed algorithm has the fastest convergence rate and the same steady state residual error with CMA.
\end{abstract}

\section{Keywords}

Blind Equalization, CMA, Momentum, Gradient Noise, Energy Steady State

\section{Introduction}

Blind equalization can compensate and track the communication channel characteristic without training sequence, which can save the communication bandwidth and improve the communication quality [1], furthermore, it can avoid the unlock of the equalizer at the same time. Recently, many experts and scholars pay attention to the theory and algorithm of blind equalization and many progresses have been achieved. In all kinds of blind equalization algorithms, CMA has simple structure and robust convergence performance [2]. However, the convergence rate of CMA is slow and the steady state residual error is big. 
Based on CMA, many improved algorithms were proposed, and MCMA is one of the improved algorithms.

Among the adaptive filtering algorithms based on stochastic gradient descent, the momentum gradient algorithm has been proved to be an effective algorithm for accelerating the convergence rate. Meanwhile, under the condition of non-convex cost function, the gradient momentum algorithm can avoid the shallow local minimum of the cost function to a certain extent, which can improve the global convergence performance [3]. However, the momentum term in the algorithm produces additional gradient noise, which results in big steady state residual error after convergence. To overcome the disadvantage of the momentum term in the algorithm, AMCMA is a compromise method between the convergence rate and the convergence precision by adjusting the momentum factor during the iterative process. The basic ideal of AMCMA is that the bigger momentum factor is set at the initial stage of the algorithm to obtain the faster convergence rate, and with the iterative process, the momentum factor is gradually decreased to obtain the lower steady state residual error. In 1992, D.L. Yang and Z.M. Liu proposed a statistic momentum LMS (SMLMS) algorithm, in which the momentum factor is designed according to the output error function, and this method improved the performance of momentum gradient algorithm. In 1998, J. B. Xu and Y. M. Wang proposed an AMCMA blind equalization based on stop-and-go algorithm, but the parameters for adjusting the momentum factor lack of theory basis [4]. The momentum CMA blind equalization proposed in [5] is a variable study step size algorithm actually, which is a MCMA blind equalization method without considering the study step size. The MCMA blind equalization proposed in [6] and [7] based on the nonlinear transformation of the transient output error of equalizer improved the convergence performance. However, there are still no theory bases of the parameters setting. Hereby, we proposed an adaptive momentum CMA algorithm controlled by energy steady state based on the analysis of momentum term in the gradient algorithm. The proposed algorithm use the energy change rate of the blind equalizer weights as the threshold to judge the steady state during the convergence process, which can take full advantage of the momentum term for accelerating the convergence rate and overcome the additional gradient noise by momentum term after convergence. The computer simulation results show the effectiveness of the proposed algorithm.

\section{The Basic Principle of CMA}

Figure 1 shows the basic principle of CMA blind equalization. Where $\boldsymbol{x}(n)$ is the input signal, $\boldsymbol{h}(n)$ is the channel impulse response, $\boldsymbol{n}(n)$ is the channel noise with Gaussian distribution commonly, $\boldsymbol{y}(n)$ is the received signal before equalization, $\boldsymbol{w}(n)$ is the weights of the blind equalizer, $\tilde{x}(n)$ is the output signal of the blind equalizer.

The essence of blind equalization is to recovery the send signal $\boldsymbol{x}(n)$ without the information of send signal and the channel $\boldsymbol{h}(n)$. According to the 


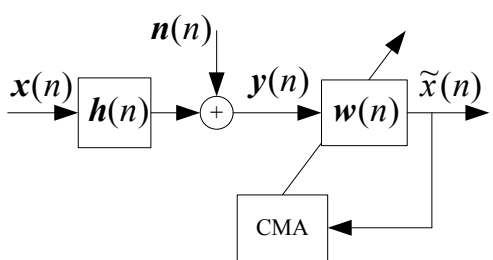

Figure 1. Block diagram of CMA blind equalization.

communication signal transmission principle, the received signal $\boldsymbol{y}(n)$ can be given by

$$
\boldsymbol{y}(n)=\boldsymbol{h}(n) \boldsymbol{x}(n)+\boldsymbol{n}(n)
$$

And the output $\tilde{x}(n)$ can be given by

$$
\tilde{x}(n)=\boldsymbol{w}^{H}(n) \boldsymbol{y}(n)
$$

CMA blind equalization utilize the higher order statistics of the transmission signal indirectly, which cost function can be given by [8]

$$
J(n)=\frac{1}{2}\left[R-|\tilde{x}(n)|^{2}\right]^{2}
$$

where $r$ is the constant modulus, which can be computed according to transmission signal as follow

$$
R=\frac{E\left[|\tilde{x}(n)|^{4}\right]}{E\left[|\tilde{x}(n)|^{2}\right]}
$$

According to stochastic gradient descent algorithm, the blind equalizer weights updating formula of CMA blind equalization can be given by [9]

$$
\boldsymbol{w}(n+1)=\boldsymbol{w}(n)+\mu e(n) \boldsymbol{y}^{*}(n) \tilde{x}(n)
$$

where $\mu$ is the study step size and $e(n)$ is the transient gradient error which can be given by

$$
e(n)=R-|\tilde{x}(n)|^{2}
$$

\section{Momentum CMA Blind Equalization}

\subsection{Adaptive Momentum CMA}

For the convergence rate of CMA blind equalization is very slow, it needs a large number of information symbols to capture the channel characteristics, which results in high bit error rate (BER) in receiver signal. Especially in high speed communication system or in time varying channel conditions, the performance of CMA blind equalization shows very poor for tracking the channel characteristics. Momentum gradient algorithm can improve the convergence rate significantly, and it can avoid falling into the shallow local minimum of the non-convex cost function to improve the global convergence ability. The momentum CMA blind equalization updates the equalizer weights by Equation (7), which a momentum term adds to the updating formula of CMA. 


$$
\begin{aligned}
\boldsymbol{w}(n+1) & =\boldsymbol{w}(n)+\mu e(n) \boldsymbol{y}^{*}(n) \tilde{x}(n) \\
& +\alpha[\boldsymbol{w}(n)-\boldsymbol{w}(n-1)]
\end{aligned}
$$

where $0<\alpha<1$ is the momentum factor which controls the contribution of momentum term in the algorithm and $\alpha[\boldsymbol{w}(n)-\boldsymbol{w}(n-1)]$ is the momentum term. If let $\Delta \boldsymbol{w}(n)=\boldsymbol{w}(n+1)-\boldsymbol{w}(n)$, then

$$
\Delta \boldsymbol{w}(n)=\mu e(n) \boldsymbol{y}^{*}(n) \tilde{x}(n)+\alpha \Delta \boldsymbol{w}(n-1)
$$

Furthermore, $\Delta \boldsymbol{w}(n)$ can be rewritten as follow

$$
\Delta \boldsymbol{w}(n)=\sum_{i=1}^{n-1} \alpha^{n-i} \nabla J(i)+\alpha^{n}(\boldsymbol{w}(1)-\boldsymbol{w}(0))
$$

where $\nabla J(n)$ is the transient gradient that is defined according to CMA cost function. To ensure the algorithm has robust convergence performance, it requires $|\alpha|<1$. If $\alpha<0$, the momentum term has no effect on the accelerating convergence of the algorithm. Therefore, the range of momentum factor is $0<\alpha<1$. When the ideal equalization condition is reached, it expects $\Delta \boldsymbol{w}(n)=\mathbf{0}$. Meanwhile, Equation (7) shows that when ideal equalization is reached, the momentum term incurs additional gradient noise, which results in big steady state residual error after the algorithm convergence.

Adaptive momentum algorithms update the weights of equalizer by the bigger momentum factor in the initial stage to accelerate the convergence rate, and the momentum factor is gradually reduced to obtain a smaller steady-state residual error in the iterative process, which can make a tradeoff between convergence rate and convergence precision. Typical adaptive momentum algorithms have the following forms

$$
\alpha(n)=\beta e(n) / \sum_{j=1}^{n} e(j)
$$

where $\beta$ is ratio coefficient as a variable parameter.

$$
\alpha(n)= \begin{cases}\frac{2}{3} & E(n) \geq 2 \\ E(n) / 3 & E(n)<2\end{cases}
$$

where $E(n)$ is mean square error which can be estimated by

$$
E(n+1)=\lambda E(n)+(1-\lambda)|\tilde{x}(n)-\hat{x}(n)|^{2}
$$

where $\lambda$ is the forgetting factor and $\hat{x}(n)$ is the decision output of $\tilde{x}(n)$.

$$
\begin{aligned}
& \alpha(n)=\gamma\left[1-e^{p-E(n)}\right] \\
& \alpha(n)=\gamma\left[1-e^{p-|e(n)|^{2}}\right]
\end{aligned}
$$

In Equation (13) and Equation (14), $\gamma$ is an adjustable parameter and $p$ is a parameter which control the positive or negative of $\alpha(n)$. Among the above adaptive momentum gradient algorithms, Equation (13) and Equation (14) have little difference. In the convergence direction of the algorithm, both $E(n)$ and $e(n)$ gradually reduce according to the statistical significance and tend to 0 . If the parameters are set appropriate, the momentum factor $\alpha(n)$ must have a 
decreasing trend and if the algorithm obtains convergence result, the following result can be obtained

$$
\lim _{n \rightarrow \infty} \alpha(n)=\gamma\left(1-e^{p}\right)
$$

Therefore, the adaptive momentum gradient algorithms according to Equation (13) and Equation (14) have the similar form with Equation (10). Furthermore, they have experience parameter $p$ needing to set and more parameters lead the algorithm to have not to be used universally. By contrast, the adaptive momentum gradient algorithm given by Equation (11) needs no parameter to be set, however, the momentum factor has no theory basis in the algorithm and the performance shows difference under different modulate signal or different SNR conditions.

\subsection{Analysis of Energy Steady State and the Algorithm Design}

Based on the analysis of momentum gradient algorithm, the momentum factor should has a bigger value to accelerate the convergence rate in the initial stage of the algorithm, when the ideal equalization is reached, the momentum factor should be 0 to avoid the additional gradient noise. A point of view was proposed in [7] that the negative momentum factor can reduce the residual error. However, there is no theoretical basis for this view. A large number of simulation results show that whether the momentum factor is positive or negative, the steady state error will increase, that is, the gradient noise will increase compared with the traditionalstochastic gradient descent algorithm.

Hereby an adaptive momentum CMA blind equalization controlled by energy steady state was proposed. According to adaptive filter theory, the weights of equalizer reach to steady state when the algorithm achieves convergence. The energy of the weights of the equalizer is defined as follow

$$
\sigma(n)=\sum_{j=1}^{L}\left|\boldsymbol{w}_{j}(n)\right|^{2}
$$

where $L$ is the length of the blind equalizer. Then the energy change rate of the weights of the blind equalizer can be defined as follow

$$
\begin{aligned}
& \Delta \sigma(n)=\sigma(n)-\sigma(n-1) \\
& \quad \quad=\sum_{j=1}^{L}\left(\left|\boldsymbol{w}_{j}(n)\right|^{2}-\left|\boldsymbol{w}_{j}(n-1)\right|^{2}\right)
\end{aligned}
$$

The energy of the weights of the blind equalizer will reach to steady state, and then the energy change rate tends to 0 . However, in the actual communication system, the received signal is often interfered by the noise, which results in the energyof the blind equalizer shows jitter downward trend. Here $\Delta \sigma(n)$ is estimated by sliding window as follow

$$
\Delta \sigma(n+1)=\lambda \Delta \sigma(n)+(1-\lambda) \Delta \sigma(n-1)
$$

where $\lambda$ is the forgetting factor. When $\Delta \sigma(n)<10^{-3}$, it can judge the algorithm has converge to the steady state. Therefore, the updating method of the momentum factor of the adaptive momentum CMA blind equalization controlled by the energy change ratio can be given by 


$$
\alpha(n)=\left\{\begin{array}{cc}
\alpha(0) & \Delta \sigma(n) \geq 10^{-3} \\
0 & \Delta \sigma(n)<10^{-3}
\end{array}\right.
$$

\section{Computer Simulation and Analysis}

To verify the performance of the adaptive momentum CMA blind equalization controlled by energy steady state, computer simulation has done to compare with the statistical momentum CMA (SMCMA) and mean square error controlled momentum CMA (MSEMCMA). Because the adaptive momentum algorithm proposed in [6] and [7] has the same sense with SMCMA and experience needs to set, here is no longer compare. In the simulation, the channel model is sparse underwater acoustic channel with two paths [10] and its transfer function can be given by

$$
H(z)=1+0.4 z^{-12}
$$

The transmission signal is equal probability binary sequence and modulate by QPSK. The noise in the channel is adding Gaussian white noise and $S N R=20 d B$. The length of the blind equalizer is 48 with the centre tap initializes to 1 and the others taps initialize to 0 . The study step size $\mu=0.001$. In SMCMA, the parameter of the momentum control function $\beta=0.75$ and the initial momentum factor $\alpha(0)=0.75$. In the estimation of the mean square error $E(n)$ and the energy change rate $\Delta \sigma(n)$, the forgetting factor $\lambda=0.99$. The initial energy change rate value of the blind equalizer is set $\Delta \sigma(0)=1$. The performance comparison conducted by the residual inter-symbol interference (ISI) [11] which defined as follow

$$
I S I(n)=\frac{\sum_{i}\left|\boldsymbol{C}_{i}(n)\right|^{2}-\left|\boldsymbol{C}_{i}(n)\right|_{\max }^{2}}{\left|\boldsymbol{C}_{i}(n)\right|_{\max }^{2}}
$$

where $\boldsymbol{C}(n)$ is the combined impulse response of the channel and the blind equalizer. The residual inter-symbol interference convergence result in the simulation achieved across 500 times Monte-Carlo is shown in Figure 2. The energy change rate of the blind equalizer vary with the iterative times is shown in Figure 3.

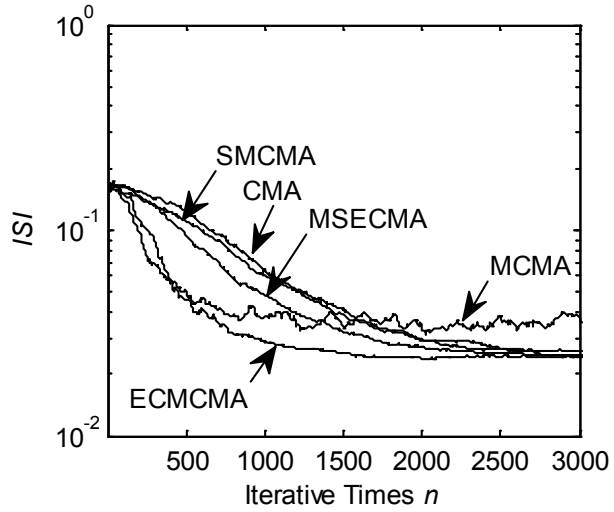

Figure 2. ISI convergence curve. 


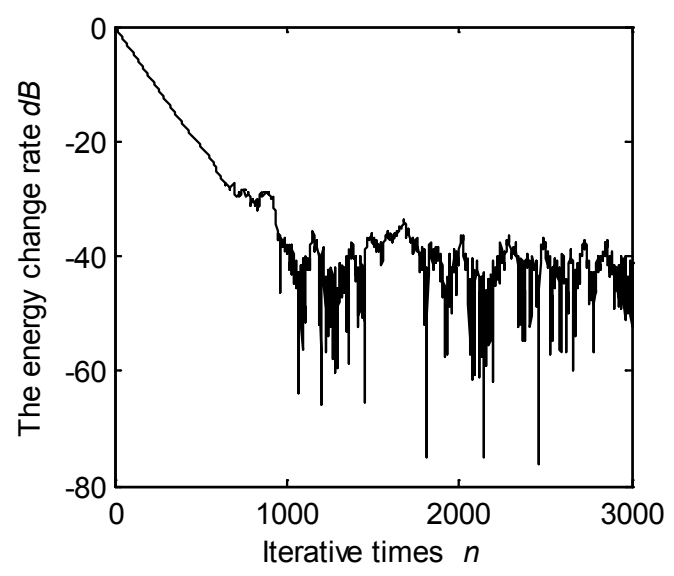

Figure 3. Energy change rate curve.

Figure 2 shows that the proposed method (ECMCMA) has the same convergence rate with the MCMA in the initial stage of the algorithm. However, ECMCMA has lower steady state residual error than MCMA after the algorithm convergence, and the steady state residual error has the same level with CMA. SMCMA and MSECMA improve the convergence rate ability is not as good as ECMCMA. Furthermore, the steady state residual error of SMCMA and MSECMA is higher than CMA. Figure 3 shows that the energy of the blind equalizer weights reaches to the steady state when the energy change rate lower than $10^{-3}$, which shows $10^{-3}$ can be taken as the criterion of the momentum factor adjustment.

The simulation results show that ECMCMA can take full advantage of the momentum term to accelerate the convergence rate. Meanwhile, it can avoid the additional gradient noise introduced by momentum term after the algorithm convergence. Also, ECMCMA can be applied with the other improved MCMA to further improve the convergence performance.

\section{Conclusion}

Momentum CMA blind equalization can improve the convergence rate and avoid the local minimum of the non-convex cost function during the iterative process. However, the additional gradient noise is introduced by momentum term after the algorithm convergence, which results in the steady state residual error increase. Adaptive momentum CMA blind equalization reduces the momentum factor during the iterative process to obtain a compromise result between convergence rate and convergence precision. This work proposed an adaptive momentum CMA blind equalization controlled by energy steady state, in which the energy change rate of the blind equalizer is defined. The threshold is set according to the energy change rate which controls the scope of action of the momentum term in the algorithm. ECMCMA takes full advantage of the momentum term to accelerate the convergence rate of the algorithm and avoid the additional gradient noise introduced by momentum term. The computer simulation results show the effectiveness of ECMCMA. 


\section{Acknowledgements}

This work was partially supported by National Science Foundation of P.R. China (Grant: 61201418), Fundamental Research Funds for the Central Universities (Grant: DC201502060302) and Liaoning Province High School Talent Support Program (Grant: LJQ2013126).

\section{References}

[1] Xu, F., Qiu, L.-D. and Wang, Y. (2013) Hybrid Blind Equalizing Method for APSK Signals. Journal of Chongqing University of Posts and Telecommunications (Natural Science Edition), 25, 605-610.

[2] Xiao, Y. and Yin, F.-L. (2014) Blind Equalization Based on RLS Algorithm Using Adaptive Forgetting Factor for Underwater Acoustic Channel. China Ocean Engineering, 28, 401-408. https://doi.org/10.1007/s13344-014-0032-5

[3] Han, Y.-G., Guo, Y.-C., Li, B.-K., et al. (2008) Momentum Term and Orthogonal Wavelet-Based Blind Equalization Algorithm. Journal of System Simulation, 20, 1559-1562.

[4] Xu, J.-B. and Wang, Y.-M. (1998) Selecting $\beta$ in "Stop and GO" Decision-Directed Algorithm and Its Momentum Algorithm. Journal of Electronics, 20, 321-329.

[5] Zhang, S.-X., Zhang, S.-Y. and Jiang, Y.-Z. (2007) Momentum Constant Modulus Algorithm for Blind Channel Equalization. Journal of Naval University of Engineering, 19, 99-102.

[6] Xing, L.-K., Wu, L. and Guo, Y.-C. (2010) Variable Momentum Factor Odd Symmetry Error FunctionBlind Equalization Algorithm. Computer Engineering and Application, 46, 117-119.

[7] Zhang, Y.-P. and Cui, W.-X. (2013) Multi-Mode Blind Equalization Algorithm with Dynamic Momentum Factors and Conjugate Gradient. Journal of Henan University of Science and Technology. Nature Science, 34, 40-44.

[8] Ning, X.-L., Liu, Z., Luo, Y.-S., et al. (2011) Improved Blind Equalization Algorithm Suitable for High-Order QAM Signals Used in Underwater Acoustic Channels. Journal of Chongqing University of Posts and Telecommunications (Natural Science Edition), 23, 516-520.

[9] Han, H.D. and Ding, Z. (2012) Steepest Descent Algorithm Implementation for Multichannel Blind Signal Recovery. IET Communication, 18, 3196-3203. https://doi.org/10.1049/iet-com.2011.0764

[10] Xiao, Y. (2015) Underwater Acoustic Channel Constant Modulus Algorithm Blind Equalization: Theory, Algorithm and Simulation. Posts \& Telecom Press, Beijing.

[11] Ruan, X.-K. and Zhang, Z.-Y. (2011) A Novel Blind Equalization Method of Complex Constellation Signals. Acta Electronica Sinica, 39, 1502-1507. 
Submit or recommend next manuscript to SCIRP and we will provide best service for you:

Accepting pre-submission inquiries through Email, Facebook, LinkedIn, Twitter, etc. A wide selection of journals (inclusive of 9 subjects, more than 200 journals)

Providing 24-hour high-quality service

User-friendly online submission system

Fair and swift peer-review system

Efficient typesetting and proofreading procedure

Display of the result of downloads and visits, as well as the number of cited articles Maximum dissemination of your research work

Submit your manuscript at: http://papersubmission.scirp.org/

Or contact ijens@scirp.org 